Original Research

\title{
Ad Libitum Western Diet Feeding Does Not Alter Basal Skeletal Muscle Heat Shock Protein Expression in Sedentary or Aerobically Trained Young Rats
}

Alex T. Von Schulze ${ }^{1,}{ }^{*}$, Marie van der Merwe ${ }^{2}$, Chad D. Touchberry ${ }^{3}$, Richard J. Bloomer ${ }^{2}$

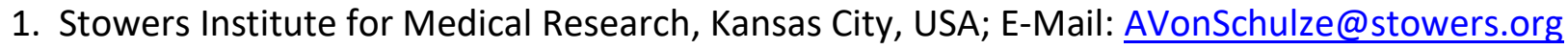

2. University of Memphis, College of Health Sciences, Roane Fieldhouse, Memphis, TN, USA; E-Mails: mvndrmrw@memphis.edu; rbloomer@memphis.edu

3. University of Missouri - Kansas City, School of Medicine, 2411 Holmes Street, Kansas City, MO, USA; E-Mail: touchberryc@umkc.edu

* Correspondence: Alex T. Von Schulze; E-Mail: AVonSchulze@stowers.org

Academic Editor: Jennifer Keogh

Special Issue: Feature Papers of Recent Progress in Nutrition 2021

Recent Progress in Nutrition

2021, volume 1 , issue 4

doi:10.21926/rpn.2104001
Received: August 03, 2021

Accepted: October 11, 2021

Published: October 15, 2021

\begin{abstract}
Poor dietary habits can lead to obesity and insulin resistance-both of which can impair basal heat shock protein (HSP) expression and the HSP stress response in skeletal muscle. It remains unclear if impairments in HSP expression occur during the early stages of diet-induced obesity and metabolic dysfunction. We determined if basal HSP expression (HSP70, HSP60, HSP25) was impaired in sedentary or exercised rats following the onset of diet-induced obesity. Male Long-Evans rats ( $N=6-7 /$ group) were assigned to a Western diet (WD) or purified diet (PD). Animals were divided into sedentary (WD and PD) or exercise-trained (WD+Ex and PD+Ex) groups and fed ad libitum for 12-weeks. WD animals displayed higher body mass, fat mass, blood glucose, and HOMA-IR scores compared to PD $(p<0.05)$. Exercise attenuated elevations in HOMA-IR and body mass for WD+Ex ( $p>0.05)$ but did not prevent elevations in fat mass or blood glucose when compared to controls. Basal HSP (HSP70, HSP60, HSP25) expression was not impaired in sedentary WD animals when compared to PD ( $p>0.05)$ or when WD+Ex was
\end{abstract}

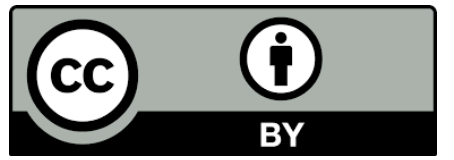

(c) 2021 by the author. This is an open access article distributed under the conditions of the Creative Commons by Attribution License, which permits unrestricted use, distribution, and reproduction in any medium or format, provided the original work is correctly cited. 
compared to PD+Ex $(p>0.05)$. Exercise training elevated HSP70 expression in the gastrocnemius muscle (GAST) $(p<0.05)$, but not in the soleus (SOL) $(p>0.05)$ in WD+Ex and PD+Ex animals. HSP60 and pHSP25 were unaffected by exercise training (GAST and SOL, $p>0.05$ ). The onset of diet-induced obesity does not impair skeletal muscle HSP expression in sedentary or exercised animals. Thus, obesity and symptomology of metabolic dysfunction may occur before reductions in skeletal muscle HSP expression.

\section{Keywords}

Exercise; skeletal muscle; heat shock proteins; obesity; high-fat diet

\section{Introduction}

Physical inactivity and poor dietary choices often lead to significant weight gain over time. Obesity is a well-known risk factor for the development of insulin resistance (IR) and type 2 diabetes (T2D). A growing body of evidence suggests that the development of obesity, IR, and T2D is closely tied to a family of molecular chaperones, known as heat shock proteins (HSP) [1, 2]. Specifically, HSP70 gene polymorphisms are associated with the development of obesity [3]. Additionally, subjects with IR and diabetes display marked reductions in basal skeletal muscle HSP expression [2]. However, findings are conflicting in high-fat-fed animal models [2]. While it remains unclear what precise effect obesity has on HSP expression, it is possible that obesity-related impairments in HSP expression may contribute to the etiology of IR and T2D. Thus, there is a strong rationale to understand how the stress of obesity can affect HSP expression.

While reduced HSP expression is tied to obesity, IR, and T2D, restoration of skeletal muscle HSP expression via heat, pharmacologic intervention, and transgenic overexpression can restore glucose tolerance and insulin sensitivity in models of high-fat feeding [4-6]. Therefore, research has focused on interventions that can improve HSP expression in skeletal muscle. Aerobic exercise is an example of an intervention that both increases skeletal muscle HSP expression and restores insulin sensitivity/metabolic function [7]. While previous investigations suggest that the HSP and metabolic responses to exercise are blunted in diabetic animals and those with a low aerobic capacity [8, 9], it is unknown if this paradigm is true during obesity. Understanding the temporal decline in HSP expression during the progression from obesity to IR and T2D is critical to establish targeted therapeutic avenues for these conditions.

Due to conflicting data within the field and a lack of knowledge regarding the temporal change in HSP expression during the progression from obesity to IR, we have taken the approach of focusing on changes in HSP expression during a state of diet-induced obesity. In addition, we have expanded our analysis to include HSP70, as well as pHSP25, HSP25, and HSP60. Our goals with the present study were two-fold: 1) determine if diet-induced obesity impairs basal expression of HSPs in skeletal muscle, and 2) determine the effects of chronic exercise training on the basal expression of HSPs in the skeletal muscle of obese animals. We hypothesized that the induction of obesity following ad libitum Western Diet (WD) feeding would lead to impaired basal HSP expression in sedentary animals. As exercise is shown to increase HSP expression [7], we hypothesized that 
exercise training would enhance the basal HSP expression in the WD-fed animals and animals fed a purified (vegan) diet (PD).

\section{Materials and Methods}

\subsection{Materials}

Cell extraction buffer (CEB) and protease inhibitor cocktail (PI) were purchased from Invitrogen (Carlsbad, CA). Phenylmethanesulphonylfluoride (PMSF), sodium orthovanadate (SOV), and sodium fluoride (NaF) were purchased from Sigma Aldrich (St. Louis, MO). Bradford protein assay reagent was purchased from Bio-Rad (Hercules, CA). 5X lane reducing sample buffer was purchased from Fisher Scientific (Pittsburgh, PA). HSP70 primary antibody (ADI-SPA-810-F) was purchased from Enzo Life Sciences (Farmingdale, NY). Phospho-HSP25 (Ser82) (\#2406), HSP25 (\#2442), and $\beta$-Tubulin (\#2146) primary antibodies, as well as Anti-rabbit IgG (\#7074) and Anti-mouse IgG (\#7076) HRPconjugated secondary antibodies, were purchased from Cell Signaling Technology (Danvers, MA). The chemiluminescent agent was purchased from Millipore (Billerica, MA). The rat insulin ELISA (80INSRT-E01) and enzymatic glucose assay reagents were purchased from Alpco (Salem, NH) and Thermo Fisher Scientific (Waltham, MA) respectively.

\subsection{Animals}

Twenty-eight male Long-Evans rats (3-4 weeks old) were obtained from Harlan Laboratories (Indianapolis, IN). Animals were housed in a climate-controlled room $\left(21^{\circ} \mathrm{C}\right)$ on a $12: 12$-h light-dark cycle (lights on at $0300 \mathrm{~h}$ ) and were allowed to acclimate for two weeks. Individual activity levels were not monitored outside of exercise training and animals did not have access to any additional training apparatuses (i.e. running wheels) for the duration of the study. All housing and experimental procedures were in accordance with the $8^{\text {th }}$ edition of the Guide for the Care and Use of Laboratory Animals and were approved by the Institutional Animal Care and Use Committee of the University of Memphis.

\subsection{Diets}

The animals were randomly assigned to one of four intervention groups; Purified Diet without exercise (PD; $n=7)$; Purified Diet with exercise (PD+Ex; $n=7)$, Western Diet without exercise (WD; $n=7)$; Western Diet with exercise (WD+Ex; $n=7)$. Upon group assignment, animals were fed $a d$ libitum and given a two-week transition period into their respective diet groups. Following the dietary transition, animals were subjected to a 12-week dietary and exercise intervention phase. $A d$ libitum feeding was chosen, as it sufficiently represents the normal feeding patterns for humans that develop metabolic syndrome. The PD (\#D13092801) and WD (\#D12079B) were purchased in pellet form from Research Diets, Inc. (New Brunswick, NJ). The PD diet was comparable to that of a standard human vegan diet (15\% protein [soy based], $60 \%$ carbohydrate [low glycemic index/high fiber], and $25 \%$ fat [primarily mono- and poly-unsaturated]). The WD diet was comparable to that of a standard human Western Diet (17\% protein [casein based], $43 \%$ carbohydrates [high glycemic index/low fiber], and $40 \%$ fat [primarily saturated]). The PD group was set as the experimental control, as it is a plant-based diet that has beneficial effects on cardio-metabolic parameters and 
antioxidant/oxidative status in human subjects [10-12]. A summary of all groups and experimental procedures is presented in Figure 1.

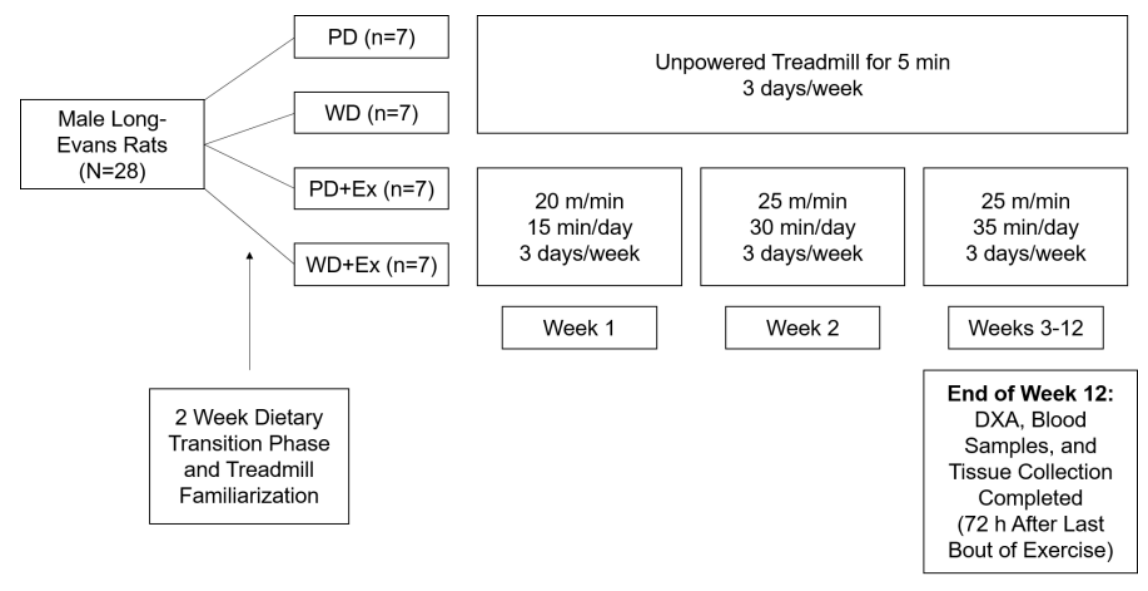

Figure 1 Summary of experimental design. All animals were sacrificed the end of the 12-week dietary and exercise intervention phase ( $72 \mathrm{~h}$ after the last bout of exercise). Food was removed from cages $2 \mathrm{~h}$ before sacrifice.

\subsection{Exercise Training \& Testing}

The current study utilized aerobic exercise, as it is a widely accepted treatment modality for obesity and IR [13], and has been shown to induce HSP expression [7]. During the two-week dietary transition period, animals in the exercise groups ( $P D+E x$ and $W D+E x)$ were familiarized with the treadmill (Exer-6M Treadmill, Columbus Instruments, Columbus, $\mathrm{OH}$ ), and exercised 3 days/week for $5 \mathrm{~min}$ at $15-20 \mathrm{~m} / \mathrm{min}$. During the 12-week dietary and exercise intervention phase, the WD+Ex and PD+Ex groups performed aerobic exercise on a treadmill 3x/week (Monday-Wednesday-Friday). During week 1, animals trained at a speed of $20 \mathrm{~m} / \mathrm{min}$ for $15 \mathrm{~min} /$ day; during week 2, $25 \mathrm{~m} / \mathrm{min}$ for $30 \mathrm{~min} /$ day; and during the remainder of the intervention phase (weeks 3-12), $25 \mathrm{~m} / \mathrm{min}$ for 35 $\mathrm{min} /$ day. The duration, intensity, and frequency of the exercise program were based on previous literature showing that treadmill running at a volume and intensity of $20 \mathrm{~m} / \mathrm{min}$ for $20 \mathrm{~min} /$ day completed 3x/week significantly improved exercise capacity after 8 weeks [14]. Animals in the sedentary groups (PD and WD) were placed on an unpowered treadmill 3x/week (MondayWednesday-Friday) for five minutes, for the duration of the study.

\subsection{Anthropometric Measurements}

Following the 12-week dietary and exercise intervention phase, all animals were subjected to a dual-energy x-ray absorptiometry (DXA) exam using a Hologic Discovery QDR series device (Hologic Inc., Bedford, MA). Animals were anesthetized with isoflurane (3\%; 1L/min O2) for the duration of the DXA examination ( $10 \mathrm{~min})$. DXA reliability was determined prior to individual examinations, having a threshold value set at $<1.5 \%$ variance between preliminary scans. Two scans were performed for each animal during the DXA examination period. If the variation between scans was greater than $1.5 \%$ an additional scan was performed. Mean values were calculated for total body mass, lean body mass, and fat mass. We defined obesity as a significant accumulation of fat mass (body fat $>30 \%$ ) over controls [15]. 


\subsection{Blood Collection, Muscle Dissection, and Tissue Storage}

After the study, the rats were euthanized ( $\mathrm{CO} 2$ inhalation) at approximately 72 hours after the last exercise bout to avoid acute responses to exercise and evaluate the adaptive responses to the treatment regimes. Food was removed from all cages ( $2 \mathrm{~h}$ before sacrifice) and blood samples were collected immediately after death from the vena cava in tubes containing ethylenediaminetetraacetic acid (EDTA). Tubes were centrifuged for $15 \mathrm{~min}$ at $1,500 \mathrm{~g}\left(4^{\circ} \mathrm{C}\right)$ to obtain plasma. Plasma samples were aliquoted into individual microtubes and stored at $-70^{\circ} \mathrm{C}$ until analysis. Upon analysis, samples were thawed and analyzed for plasma glucose and insulin levels. The soleus (SOL) and gastrocnemius (GAST) were surgically removed, immediately frozen in liquid nitrogen, and stored at $-70^{\circ} \mathrm{C}$ until analysis.

\subsection{Blood Glucose and Insulin}

Plasma glucose and insulin were quantified using commercially available assay kits/reagents. All procedures were performed per the manufacturer's instructions and sample values were determined using a BioTek PowerWave Microplate Spectrophotometer (Winooski, VT). Following previous literature, we deemed hyperglycemia as a plasma glucose value $>150 \mathrm{mg} / \mathrm{dL}$ [16]. The metabolic function was inferred using the HOMA-IR [17]. The HOMA-IR was scaled to fit a rodent model and calculated as (Plasma Glucose $[\mathrm{mg} / \mathrm{dL}] \times$ Plasma Insulin $[\mu \mathrm{U} / \mathrm{mL}]$ )/2,658 as described previously [18].

\subsection{Protein Extraction, Protein Quantification, and Western Blotting}

GAST and SOL segments were cut on dry ice (50 mg pieces) and homogenized in a 12:1 (volume/weight) CEB solution containing PI, 200mM PMSF, 200mM Na $\mathrm{mO}_{4}$, and 200mM $\mathrm{NaF}$. Samples were put into individual microtubes, placed on ice, and homogenized in Kontes glass, and then vortexed (every $10 \mathrm{~min}$ for $30 \mathrm{~min}$ ). Samples were then centrifuged for $10 \mathrm{~min}$ at 3,000 g. Total protein concentration was assessed using the Bradford method [19]. Sample concentrations were corrected to $8 \mu \mathrm{g} / \mu \mathrm{L}$ in $5 \mathrm{X}$ Lane Marking Reducing Sample Buffer and then heated at $95^{\circ} \mathrm{C}$ for $5 \mathrm{~min}$. Samples ( $60 \mu \mathrm{g} /$ lane) were subjected to sodium dodecyl sulfate-polyacrylamide gel electrophoresis (SDS-PAGE, 10\% gel) [20]. Following SDS-PAGE, gels were cut into multiple strips (based on protein size) to fit on the same polyvinylidene fluoride (PVDF) membrane [21], and proteins were transferred for $90 \mathrm{~min}$ ( $250 \mathrm{~mA}$ ). Membranes were blocked with 5\% non-fat dry milk (NFDM) in Trisbuffered saline-0.1\% Tween 20 (TBST) for $60 \mathrm{~min}$. Samples were then incubated overnight at $4^{\circ} \mathrm{C}$ with the appropriate primary antibodies (diluted 1: 1,000 in 5\% BSA-TBST). All blots were washed and exposed to the appropriate HRP-conjugated secondary antibody (1:10,000 in 2\% NFDM-TBST) for $90 \mathrm{~min}$ at room temperature. Protein bands were visualized with a chemiluminescent agent and imaged using a Fotodyne ${ }^{\circledR}$ 60-7020 (FOTODYNE Incorporated, Hartland, WI) benchtop image and gel documentation system. All bands were quantified 5 times via densitometry (AlphaEaseFC ${ }^{\text {TM }}$, San Leandro, CA, USA) and mean values were analyzed. Mean values for all groups were normalized to $\beta$-Tubulin. When necessary, blots were stripped with Restore ${ }^{\mathrm{TM}}$ Western Blot Stripping Buffer (Thermo Fisher Scientific, Waltham, MA) for $30 \mathrm{~min}$ at $37^{\circ} \mathrm{C}$, blocked with $5 \%$ NFDM-TBST (60 $\mathrm{min}$ ), and re-probed with the appropriate primary antibody. HSP70, HSP60, pHSP25 were chosen as they have been previously associated with obesity and insulin resistance [1, 2, 22-24]. 


\section{Statistical Analysis}

All statistical procedures were performed using GraphPad Prism $6^{\circledR}$ software (GraphPad Software Inc., La Jolla, CA). All data are presented as mean \pm SEM. Statistical significance was set at $\alpha=0.05$. Mean values for blood markers of metabolic function, anthropometric measures, and HSP expression were analyzed by two-way analysis of variance (ANOVA) and follow-up t-tests using the modified Holm's sequential Bonferroni method [25]. A Pearson product-moment correlation coefficient $(r$ ) was performed to determine if there was a correlation between anthropometric data and HOMA-IR, HSP expression and HOMA-IR, and HSP expression and fat mass.

\section{Results}

A total of 27 animals completed all aspects of this study. One rat in the WD+Ex group died during week two of the intervention, approximately $30 \mathrm{~min}$ after an exercise session. The necropsy revealed the abdomen was filled with blood, with the likely cause of death a suspected aneurism. All other animals completed the 12-week intervention.

\subsection{Plasma Glucose and HOMA-IR}

Significant main effects were observed for diet and exercise when comparing plasma glucose values after the intervention phase $(p<0.05)$. However, there was no interaction between diet and exercise when comparing plasma glucose values. Plasma glucose was significantly higher in the WD $(+41.15 \%)$ and WD+Ex (+40.21\%) groups when compared to the PD and PD+Ex controls $(p<0.05$, Figure 2a). Also, the WD group had significantly higher plasma glucose (+61.65\%) compared to the $P D+E x$ group $(p<0.05$, Figure $2 a)$. No significant variations in plasma glucose were noted between $P D$ and $P D+E x$, nor between WD and WD+Ex (Figure 2a). Plasma glucose values revealed that the WD $(182.50 \pm 18.27 \mathrm{mg} / \mathrm{dL})$ and WD+Ex $(158.30 \pm 24.39 \mathrm{mg} / \mathrm{dL})$ groups developed hyperglycemia (plasma glucose $>150 \mathrm{mg} / \mathrm{dL}$ ), while the PD and PD+Ex did not develop hyperglycemia $(129.00 \pm 22.86 \mathrm{mg} / \mathrm{dL}$ and $112.90 \pm 7.19 \mathrm{mg} / \mathrm{dL}$ respectively). Plasma insulin for the WD, WD+Ex and $\mathrm{PD}+$ Ex groups were not significantly different when compared to the PD control group (Figure $2 \mathrm{~b}$ ). When comparing HOMA-IR scores, the ANOVA revealed a significant main effect of diet on the HOMA-IR score $(p<0.05)$. Follow-up tests showed significant elevations in the degree of HOMA-IR for the WD group compared to the PD and PD+EX groups $(+316.33 \%$ and $+249.00 \%$ respectively, $\mathrm{p}<0.05$, Figure $2 \mathrm{c}$ ). Exercise did not significantly affect HOMA-IR scores within dietary conditions (PD vs $P D+E x$ and $W D$ vs WD+Ex) (Figure $2 c$ ) but did attenuate the degree of HOMA-IR in the WD+Ex group compared to the PD group ( $p<0.05$, Figure $2 c$ ). Finally, no significant interaction was observed between diet and exercise when comparing HOMA-IR scores. 


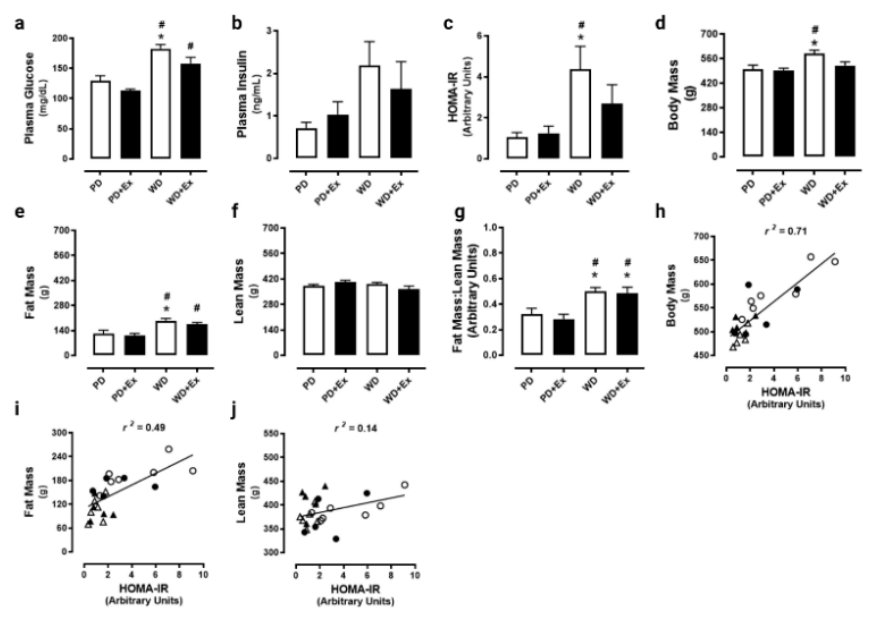

Figure 2 12-week ad libitum feeding of WD induces obesity and elevates surrogate markers of metabolic dysfunction. a) Plasma glucose levels were unaffected in animals consuming the PD; however, animals consuming the WD had elevated levels of fasting plasma glucose. b) Fasting plasma insulin did not change significantly across the assigned groups. c) A WD induced a significant increase in HOMA-IR score, which was attenuated with exercise. d) Ad libitum WD feeding caused significant increases in body mass for sedentary animals. e) Fat mass increased substantially in WD-fed animals, and exercise was not able to significantly reduce the accumulation of fat mass. f) Lean mass was not affected by diet or exercise. g) Fat mass to lean mass ratios were significantly higher in both WD and WD+Ex groups. $h$ ) There was a large correlation between body mass and HOMA-IR. i) There was a moderate correlation between fat mass and HOMA-IR. j) There was a weak correlation between lean mass and HOMA-IR. Data are shown as mean \pm SEM, as well as linear regression line and $r^{2}$ ( $n=5-7$ samples/group). *indicates $p<0.05$ compared to the PD control, \# indicates $\mathrm{p}<0.05$ compared to the PD+Ex control.

\subsection{Body Mass, Fat Mass, Time to Exhaustion}

A significant main effect of diet was observed when comparing body mass after the 12-week dietary and exercise intervention phase $(p<0.05)$. Follow-up tests revealed that body mass was significantly higher in the WD group compared to the PD and PD+Ex controls $(+16.24 \%$ and $+14.05 \%$ respectively, $p<0.05$, Figure $2 d$ ). Exercise attenuated elevations in body mass for the WD+Ex group (WD+Ex vs PD, Figure 2d). PD and PD+Ex groups did not differ when comparing body mass (Figure $2 d$ ). Fat mass was significantly elevated in the WD and WD+Ex groups compared to the respective $\mathrm{PD}$ and $\mathrm{PD}+$ Ex controls ( $+58.56 \%$ and $+55.95 \%$ respectively, $\mathrm{p}<0.05$, Figure $2 \mathrm{e}$ ). Exercise did not significantly affect fat mass within dietary conditions (PD vs PD+Ex and WD vs WD+Ex). The lean mass remained consistent between all groups (Figure 2f). Fat mass to lean mass ratios were significantly higher in both WD and WD+Ex groups ( $p<0.05$, Figure $2 \mathrm{~g})$. Body mass, fat mass, and lean mass were positively correlated to HOMA-IR $\left(r^{2}=0.71, r^{2}=0.49\right.$, and $r^{2}=0.14$ respectively, $p<0.05$, Figure $2 \mathrm{~h}-\mathrm{j}$ ). Regular exercise training improved treadmill time to exhaustion in the PD+Ex and WD+Ex groups by $80.5 \%$ and $36.1 \%$, respectively (data not shown). Conversely, we noted a 
worsening of performance in the sedentary PD and WD groups by $-14.3 \%$ and $-14.9 \%$, respectively (data not shown).

\subsection{Basal HSP Expression and the HSP Response to Exercise}

A significant main effect of exercise was observed when comparing HSP70 expression in the GAST $(p<0.05)$. Specifically, HSP70 expression was significantly higher in the PD+Ex and WD+Ex groups compared to their respective PD and WD groups ( $+95.78 \%$ and $+93.84 \%$ respectively, $p<0.05$, Figure 3a). Additionally, HSP70 expression was significantly higher when comparing the PD and WD+Ex groups, as well as the WD and the PD+Ex groups $(+120.54 \%$ and $+72.08 \%$ respectively, $p<0.05$, Figure 3a). Exercise did not affect HSP60 and pHSP25 expression in the GAST (Figure 3b and 3c). Diet did not have any effect on basal or exercise-induced HSP expression in the GAST (Figure 3a-c). We did not observe an interaction between diet and exercise when comparing HSP expression levels in the GAST. There was no correlation between HSP70, HSP60, or PHSP25 expression and HOMA-IR in the GAST $\left(r^{2}=0.00, r^{2}=0.03\right.$, and $r^{2}=0.02, p>0.05$, Figure 4a-c respectively). Additionally, there was no correlation between HSP70, HSP60, or pHSP25 expression and fat mass in the GAST $\left(r^{2}=0.00\right.$, $r^{2}=0.00$, and $r^{2}=0.00, p>0.05$, data not shown).
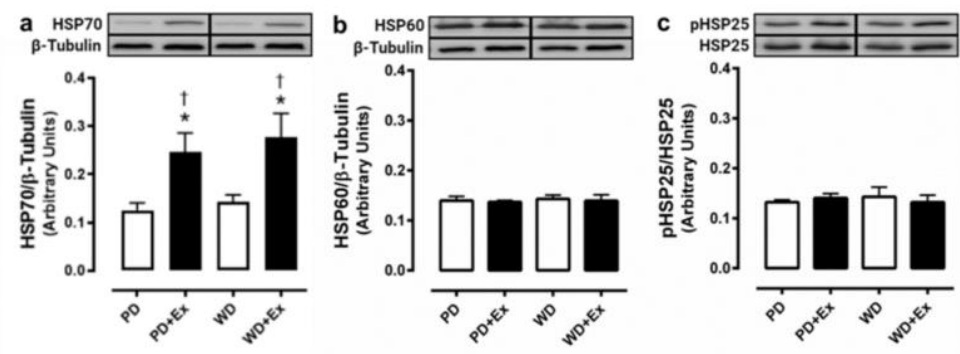

Figure 3 HSP70 expression in GAST increases with exercise but is unaffected by diet. a) HSP70 expression increased as a result of exercise; however, there were no differences in expression between dietary interventions. b) HSP60 expression and c) pHSP25/HSP25 expression were not affected by diet or exercise. Data are shown as mean \pm SEM ( $n=6$ 7 samples/group). ${ }^{*}$ indicates $p<0.05$ compared to the PD control, tindicates $p<0.05$ compared to the WD group.
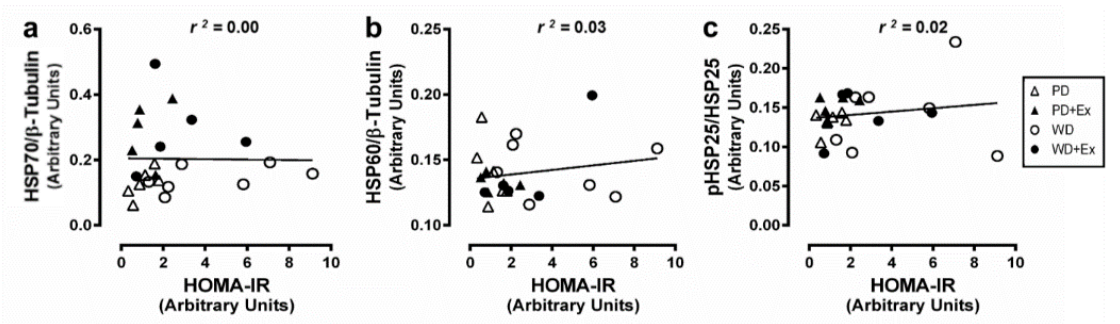

Figure 4 HSP expression in the GAST does not correlate with HOMA-IR. Pearson productmoment coefficients $(r)$ were calculated for all animals. There was no correlation between HOMA-IR and basal HSP70 (a), HSP60 (b), or pHSP25 (c) expression. Data are reported as linear regression line and $r^{2}(n=5-7$ animals/group). 
Neither exercise nor diet affected HSP70, HSP60, and pHSP25 expression in the SOL (Figure 5ac). We did not observe an interaction between diet and exercise when comparing HSP expression levels in the SOL. There was no correlation between HSP70 or HSP60 expression and HOMA-IR in the SOL $\left(r^{2}=0.02\right.$ and $r^{2}=0.00$ respectively, Figure $6 a$ and $\left.6 b\right)$. However, there was a moderate positive correlation between pHSP25 expression and HOMA-IR within the SOL $\left(r^{2}=0.32\right.$, Figure $\left.6 c\right)$. There was no correlation between fat mass and HSP expression in the SOL (HSP70: $r^{2}=0.01$, HSP60: $r^{2}=0.00$, and pHSP25: $r^{2}=0.11$, data not shown).
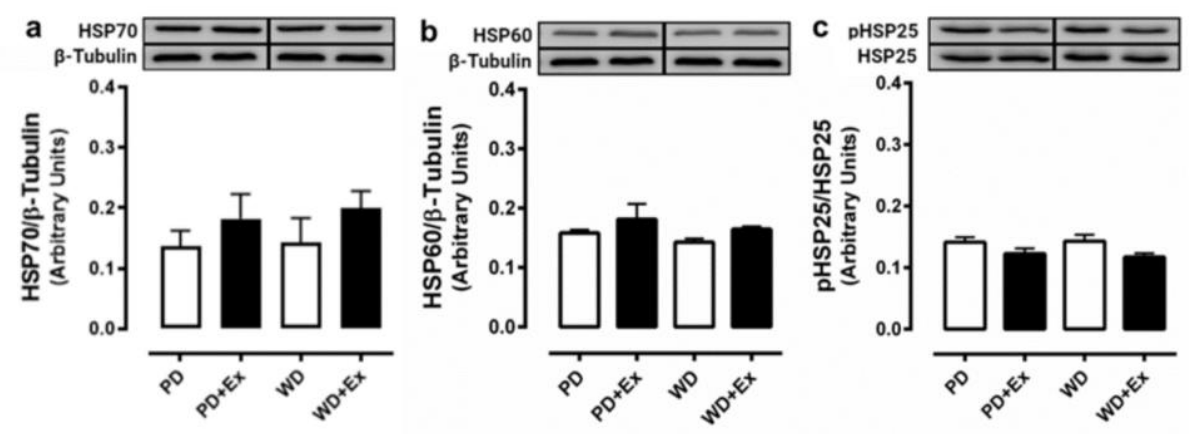

Figure 5 HSP expression in SOL was unaffected by diet and exercise. a) HSP70, b) HSP60, and c) pHSP25/HSP25 expression were not affected by diet or exercise. Data are shown as means \pm SEM ( $n=6-7$ samples/group).
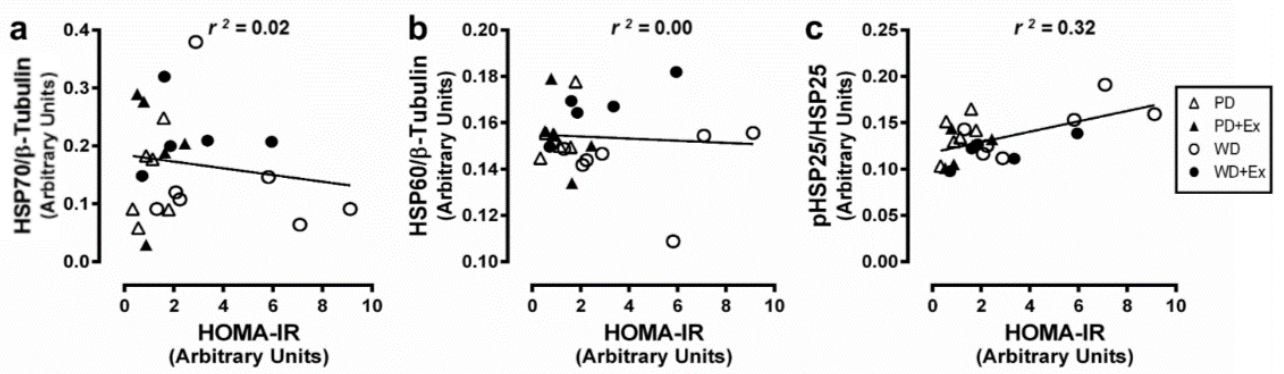

Figure 6 HSP expression in the SOL muscle was not correlated with HOMA-IR. Pearson product-moment coefficients $(r)$ were calculated for all animals. There was no correlation between HOMA-IR and basal HSP70 (a), HSP60 (b), or pHSP25 (c) expression. Data are reported as linear regression line and $r^{2}$ ( $n=5-7$ animals/group).

\section{Discussion}

Previous studies have reported contradictory results relating to basal skeletal muscle HSP70 content during states of high-fat feeding, obesity, and IR [2]. The differences between these studies may be related, in part due to the models employed, the muscle analyzed, the duration of feeding, and/or the degree of obesity. The goals of this study were to determine if basal skeletal muscle HSP expression was impaired in sedentary or aerobically exercised animals following the onset of dietinduced obesity. 
The novel findings of this study are that: 1) basal skeletal muscle HSP70, HSP60, and pHSP25 expression are not significantly altered during the onset of diet-induced obesity, and 2) onset of obesity does not attenuate the exercise-induced increase in basal HSP70 expression of the GAST. Importantly, we show that obesity and early symptomology of metabolic dysfunction can occur without impairment of basal skeletal muscle HSP expression. This suggests that impairments in basal muscle HSP expression may be a sign of more advanced metabolic disease. Further, we show that obese animals can increase basal HSP70 expression similar to control animals, which may offer them temporary protection from stressors associated with metabolic dysfunction.

\subsection{Effects of a Western Diet on Blood Biomarkers and Muscle HSP Expression}

As expected, we found that consumption of an ad libitum WD leads to obesity (elevated body mass and fat mass), hyperglycemia, and elevated HOMA-IR in our rodent model. However, these whole-body metabolic changes did not correlate with alterations in basal HSP70, HSP60, and pHSP25 expression in the SOL or GAST muscle. This was surprising, as the mean values for plasma insulin (WD $\sim 360 \mathrm{pmol} / \mathrm{L}$ and $W D+E x \sim 290 \mathrm{pmol} / \mathrm{L}$ ) and plasma glucose (WD $\sim 9.99 \mathrm{mmol} / \mathrm{L}$ and WD+Ex $\sim 8.88 \mathrm{mmol} / \mathrm{L}$ ) within our WD model were comparable to previous reports suggesting impairments of the HSP70 response in models fed a high-fat diet [4].

In agreement with prior work [2], the current study also demonstrated no significant reduction in HSP expression despite measures of metabolic dysfunction. Specifically, our HSP70 and pHSP25 data in obese rodents are consistent with experimental data in the SOL and extensor digitorum longus muscles of IR rodents [5, 6]. In both studies by Gupte et al. (2009a-b), there were no changes in basal HSP expression (HSP70, pHSP25), despite evidence of significant skeletal muscle insulin resistance. Despite null changes in basal HSP expression (HSP70, pHSP25), Gupte et al. (2009a-b) demonstrated that HSP induction (HSP70, pHSP25), by lipoic acid and hyperthermia were associated with improved insulin sensitivity. Whether these improvements in insulin sensitivity were due to HSP70 or HSP25 specifically remains to be elucidated. Despite only moderate metabolic alterations in our obese rodents, our data are also consistent with other investigations utilizing streptozotocin (STZ)-induced diabetes that documents no reductions in basal HSP70 expression for the red gastrocnemius up to 8-weeks following STZ-injection [8]. It is possible that by using a "healthy" PD, exercise-induced expression of HSPs was blunted in both dietary conditions - therefore we missed the "blunting" effect of obesity. However, we believe this is unlikely, as the degree of change in HSP70 in the GAST muscle for the PD+Ex is comparable to previous work using a standard chow diet [26].

Interestingly, our data and those mentioned above vary from others who have documented reductions in basal HSP70 expression for the vastus lateralis muscle in IR and diabetic models [2, 8]. The differences between findings are likely due to several important factors, including muscle fiber type and time under metabolic stress or severity of the metabolic disease. Basal HSP expression is known to be higher in slow-twitch muscles with a greater oxidative capacity than fast-twitch muscles [27] but it is difficult to compare the mixed fibers of the vastus lateralis to the mixed fibers in the gastrocnemius across these studies. Additionally, time under metabolic stress also appears to be a likely factor. Work in primates shows impairments in basal skeletal muscle HSP70 expression do not develop over months but do occur with years of high-fat feeding [28]. Further, glucose intolerance and obesity occur well before the onset of mitochondrial dysfunction, which takes 4- 
times longer to develop in response to high-fat feeding in mice [29]. The eventual HSP70 decline may be linked to this dysfunction, as it is shown to be critical for enhanced mitochondrial oxidation, number, and quality [30,31]. Therefore, the ability to maintain basal HSP70 expression seems to be a critical element in preserving mitochondrial function and preventing the transition from IR to T2D. It is tempting to speculate this is due to the anti-inflammatory effects of HSPs. Specifically, HSP70 is known to inhibit the pro-inflammatory c-jun terminal kinase (JNK) which amplifies mitochondrialderived reactive oxygen species upon activation [32-36].

We suggest a paradigm in which transitory changes in HSP expression occur long after the development of obesity, and may be more closely tied to the onset of T2D. Finally, to our knowledge, we are the first to study HSP60 expression in skeletal muscle during a WD intervention. We were surprised by the lack of change in skeletal muscle HSP60 expression during WD treatment, as HSP60 is a known marker of mitochondrial stress and is shown to be elevated in the serum of patients with T2Ds [37]. Future studies should explore the role of HSP60 in models of IR and T2D, particularly the effects of HSP60 overexpression.

\subsection{Effects of a Western Diet on Chronic Aerobic Exercise-Induced HSP Expression}

We observed that chronic aerobic exercise yielded a mild, non-significant protective effect on HOMA-IR and body mass, as well as markedly increased basal HSP70 expression in the GAST muscle. However, our analysis revealed that this "protective" effect from exercise was not correlated to basal HSP70 expression in the GAST muscle. Therefore, it's likely that the positive effects of exercise may have been a result of increased energy expenditure, enhanced insulin-independent glucose uptake, and/or improved skeletal muscle insulin sensitivity [13, 38, 39].

We did not observe an increase in HSP70 expression from exercise training in the SOL, contrasting others showing that HSP70 expression increases in the SOL $24 \mathrm{~h}$ following a single treadmill run (15$27 \mathrm{~m} / \mathrm{min}$ ) [40]. There are a few reasons why we may not have seen an increase in SOL HSP70 expression in our study. Previous data show that trained rodents exhibit a diminished HSP70 response in the SOL to customary exercise, because of greater antioxidant protection from training [41]. Similar patterns of an attenuated exercise-induced HSP70 response have been reported in well-trained humans [42]. In addition, exercise-induced HSP70 expression is shown to be intensitydependent [40] and our animals possibly ran at a greater relative intensity, leading to altered muscle loading and recruitment patterns. This would shift muscle recruitment towards the fast-twitch fibers in the GAST to complete the exercise training. A shift in relative intensity possibly amplified whole-body metabolic effects on insulin resistance in WD+Ex groups, as moderate to high-intensity exercise results in greater improvements, and persistence, in insulin sensitivity compared with lowintensity exercise in obese individuals [43-45]. In addition, fast-twitch muscle fibers are primarily glycolytic and thus become insulin resistant faster than slow-twitch fibers [46]. Thus, their continued activation could in part explain the amplified benefits of moderate to high-intensity exercise on insulin sensitivity. All these factors complement our data, showing that chronic exercise increased HSP70 in the GAST of the WD+Ex animals, as well as results in improved their HOMA-IR scores.

We did not observe changes in the other HSPs measured. Specifically, we found that our moderate-intensity exercise intervention did not elevate HSP60 and pHSP25 in the GAST or SOL. It is possible that our intervention was not intense enough to exhibit an increase in HSP60 [47]. 
Additionally, it is possible that HSP25 expression, and phosphorylation, are more responsive to conditions of muscle injury induced by eccentric contractions $[48,49]$.

The onset of obesity and hyperglycemia in our study did not blunt the basal HSP70 expression due to exercise training in the GAST. Recent evidence suggests that aerobic capacity may mediate the impairment of the HSP70 response in animals subjected to a high-fat diet [9]. Rogers et al. (2016) showed that rodents selectively bred to have a low aerobic capacity begin to develop signs of metabolic dysfunction and display impairments in the HSP70 response to heat shock after 3 days of high-fat feeding when compared to rodents selected for high aerobic capacity. As we were principally concerned with changes in basal HSP expression, we did not test the acute exerciseinduced HSP response in our rats. Therefore, perhaps obese animals would have an impaired HSP response to a single bout of stress (exercise or heat). Nevertheless, our data show that the enhanced aerobic capacity of our exercise-trained rodents was observed in combination with increased basal HSP70 expression in the GAST of the PD+Ex and WD+Ex groups. Thus, it may be possible for obese subjects to maintain HSP70 expression despite the onset of metabolic dysfunction if aerobic capacity is maintained.

Findings specific to the role of exercise and HSP expression are important, as obese and insulinresistant subjects may be prone to mitochondrial dysfunction [50]. It seems reasonable to determine if chronic exercise is an effective means to maintain muscle HSP expression in obese subjects, which in turn could promote better mitochondrial function and ward off metabolic disease. Thus, future studies are warranted to determine the relationship between aerobic capacity, HSP expression, and mitochondrial function in models of metabolic disease.

\section{Conclusions}

In summary, our findings show that the onset of diet-induced obesity does not impair basal HSP (HSP70, HSP60, pHSP25) expression in the SOL and GAST. In addition, obese rodents can increase basal HSP70 expression in response to chronic exercise training in the GAST muscles like their nonobese counterparts. Our data do not support our hypothesis that basal skeletal muscle HSP expression is impaired during the onset of obesity and hyperglycemia in sedentary or exercised animals. An examination of the literature suggests that declines in HSP expression, as well as deficiencies in the HSP response, during IR and T2D may be a result of the time spent under metabolic strain and/or the severity of the metabolic disease. While the literature seems clear that HSP induction improves insulin sensitivity [1, 2], further studies are needed to elucidate the molecular mechanisms by which HSP induction improves glycemic control. Lastly, it is critical to determine when skeletal muscle HSP impairment occurs on the continuum from obesity to IR and from IR to T2D. Understanding the temporal decline in HSP expression and induction during the progression of metabolic dysfunction is critical to establish targeted therapeutic avenues for these conditions.

\section{List of Abbreviations}

Heat shock protein (HSP)

Western diet (WD)

Purified diet (PD)

Homeostatic Model Assessment for Insulin Resistance (HOMA-IR) 


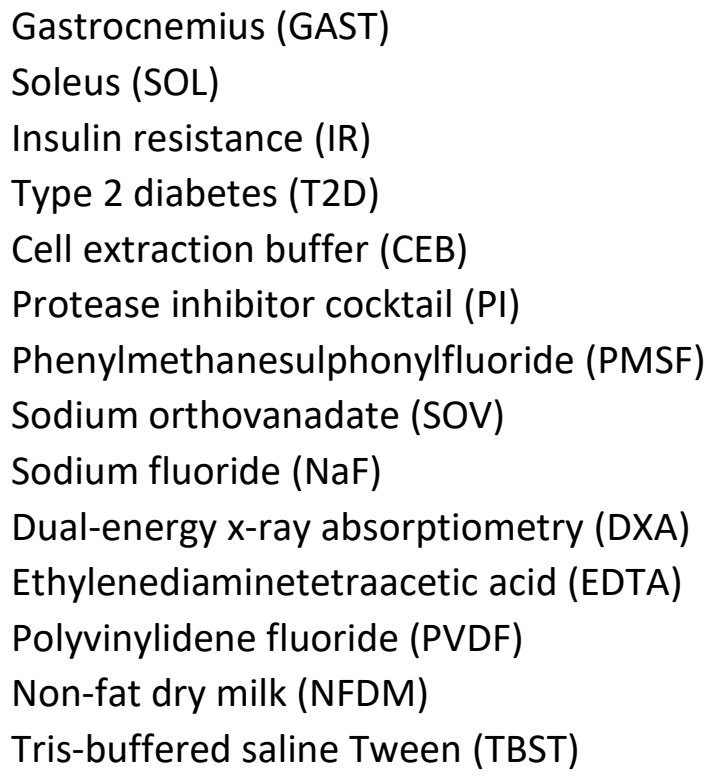

\section{Acknowledgements}

This work was funded by The School of Health Studies to Dr. Chad Touchberry while employed at The University of Memphis. Appreciation is extended to Drs. Trint Gunnels, Sang-Rok Lee, Randal Buddington, and Karyl Buddington, as well as Johnhenry Schriefer, for assistance in data collection.

\section{Author Contributions}

ATV, CDT, and RJB were responsible for the study design and oversight. MV was responsible for assistance with study design, testing, and data collection. ATV and CDT were responsible for biochemical analyses, statistical analyses, and manuscript preparation. All authors assisted with manuscript editing.

\section{Funding}

Funding for this work was provided by the University of Memphis.

\section{Competing Interests}

The authors declare no conflicts of interest related to this work.

\section{References}

1. Geiger PC, Gupte AA. Heat shock proteins are important mediators of skeletal muscle insulin sensitivity. Exerc Sport Sci Rev. 2011; 39: 34-42.

2. Archer AE, Von Schulze AT, Geiger PC. Exercise, heat shock proteins and insulin resistance. Philos Trans R Soc B Biol Sci. 2018; 373: 20160529.

3. Mardan-Nik M, Pasdar A, Jamialahmadi K, Avan A, Mohebati M, Esmaily H, et al. Association of heat shock protein70-2 (HSP70-2) gene polymorphism with obesity. Ann Hum Biol. 2016; 43: 542-546. 
4. Chung J, Nguyen AK, Henstridge DC, Holmes AG, Chan MH, Mesa JL, et al. HSP72 protects against obesity-induced insulin resistance. Proc Natl Acad Sci U S A. 2008; 105: 1739-1744.

5. Gupte AA, Bomhoff GL, Swerdlow RH, Geiger PC. Heat treatment improves glucose tolerance and prevents skeletal muscle insulin resistance in rats fed a high-fat diet. Diabetes. 2009; 58: 567-578.

6. Gupte AA, Bomhoff GL, Morris JK, Gorres BK, Geiger PC. Lipoic acid increases heat shock protein expression and inhibits stress kinase activation to improve insulin signaling in skeletal muscle from high-fat-fed rats. J Appl Physiol. 2009; 106: 1425-1434.

7. Henstridge DC, Febbraio MA, Hargreaves M. Heat shock proteins and exercise adaptations. Our knowledge thus far and the road still ahead. J Appl Physiol. 2016; 120: 683-691.

8. Atalay M, Oksala NK, Laaksonen DE, Khanna S, Nakao C, Lappalainen J, et al. Exercise training modulates heat shock protein response in diabetic rats. J Appl Physiol. 2004; 97: 605-611.

9. Rogers RS, Morris EM, Wheatley JL, Archer AE, McCoin CS, White KS, et al. Deficiency in the heat stress response could underlie susceptibility to metabolic disease. Diabetes. 2016; 65: 3341-3351.

10. Bloomer RJ, Kabir MM, Canale RE, Trepanowski JF, Marshall KE, Farney TM, et al. Effect of a 21 day Daniel Fast on metabolic and cardiovascular disease risk factors in men and women. Lipids Health Dis. 2010; 9: 1-9.

11. Bloomer RJ, Kabir MM, Trepanowski JF, Canale RE, Farney TM. A 21 day Daniel fast improves selected biomarkers of antioxidant status and oxidative stress in men and women. Nutr Metab. 2011; 8: 1-9.

12. Trepanowski JF, Kabir MM, Alleman Jr RJ, Bloomer RJ. A 21-day Daniel fast with or without krill oil supplementation improves anthropometric parameters and the cardiometabolic profile in men and women. Nutr Metab. 2012; 9: 1-7.

13. Goodpaster BH, Katsiaras A, Kelley DE. Enhanced fat oxidation through physical activity is associated with improvements in insulin sensitivity in obesity. Diabetes. 2003; 52: 2191-2197.

14. Costa MS, Ardais AP, Fioreze GT, Mioranzza S, Botton PH, Portela LV, et al. Treadmill running frequency on anxiety and hippocampal adenosine receptors density in adult and middle-aged rats. Prog Neuro-Psychopharmacol Biol Psychiatry. 2012; 36: 198-204.

15. Li S, Zhang HY, Hu CC, Lawrence F, Gallagher KE, Surapaneni A, et al. Assessment of diet-induced obese rats as an obesity model by comparative functional genomics. Obesity. 2008; 16: 811818.

16. Nauck MA, Walberg J, Vethacke A, El-Ouaghlidi A, Senkal M, Holst JJ, et al. Blood glucose control in healthy subject and patients receiving intravenous glucose infusion or total parenteral nutrition using glucagon-like peptide 1. Regul Pept. 2004; 118: 89-97.

17. Matthews DR, Hosker JP, Rudenski AS, Naylor BA, Treacher DF, Turner RC. Homeostasis model assessment: Insulin resistance and $\beta$-cell function from fasting plasma glucose and insulin concentrations in man. Diabetologia. 1985; 28: 412-419.

18. Cacho J, Sevillano J, de Castro J, Herrera E, Ramos MP. Validation of simple indexes to assess insulin sensitivity during pregnancy in Wistar and Sprague-Dawley rats. Am J Physiol Endocrinol Metab. 2008; 295: E1269-E1276.

19. Bradford MM. A rapid and sensitive method for the quantitation of microgram quantities of protein utilizing the principle of protein-dye binding. Anal Biochem. 1976; 72: 248-254. 
20. Laemmli UK. Cleavage of structural proteins during the assembly of the head of bacteriophage T4. Nature. 1970; 227: 680-685.

21. Aksamitiene E, Hoek JB, Kholodenko B, Kiyatkin A. Multistrip Western blotting to increase quantitative data output. Electrophoresis. 2007; 28: 3163-3173.

22. Tavallaie S, Rahsepar AA, Abdi H, Moohebati M, Moodi F, Pourghadamyari $H$, et al. Association between indices of body mass and antibody titers to heat-shock protein-27 in healthy subjects. Clin Biochem. 2012; 45: 144-147.

23. Märker T, Sell H, Zilleßen P, Glöde A, Kriebel J, Ouwens DM, et al. Heat shock protein 60 as a mediator of adipose tissue inflammation and insulin resistance. Diabetes. 2012; 61: 615-625.

24. Ghayour-Mobarhan M, Taylor A, Lamb DJ, Ferns GA. Association between indices of body mass and antibody titres to heat-shock protein-60, -65 and -70 in healthy Caucasians. Int J Obes (Lond). 2007; 31: 197-200.

25. Holm S. A simple sequentially rejective multiple test procedure. Scand J Stat. 1979; 6: 65-70.

26. Skidmore R, Gutierrez JA, Guerriero Jr V, Kregel KC. HSP70 induction during exercise and heat stress in rats: Role of internal temperature. Am J Physiol. 1995; 268: R92-R97.

27. Locke M, Noble EG, Atkinson BG. Inducible isoform of HSP70 is constitutively expressed in a muscle fiber type specific pattern. Am J Physiol. 1991; 261: C774-C779.

28. Kavanagh K, Wylie AT, Chavanne TJ, Jorgensen MJ, Voruganti VS, Comuzzie AG, et al. Aging does not reduce heat shock protein 70 in the absence of chronic insulin resistance. J Gerontol Ser A. 2012; 67: 1014-1021.

29. Bonnard C, Durand A, Peyrol S, Chanseaume E, Chauvin MA, Morio B, et al. Mitochondrial dysfunction results from oxidative stress in the skeletal muscle of diet-induced insulin-resistant mice. J Clin Invest. 2008; 118: 789-800.

30. Drew BG, Ribas V, Le JA, Henstridge DC, Phun J, Zhou Z, et al. HSP72 is a mitochondrial stress sensor critical for Parkin action, oxidative metabolism, and insulin sensitivity in skeletal muscle. Diabetes. 2014; 63: 1488-1505.

31. Henstridge DC, Bruce CR, Drew BG, Tory K, Kolonics A, Estevez E, et al. Activating HSP72 in rodent skeletal muscle increases mitochondrial number and oxidative capacity and decreases insulin resistance. Diabetes. 2014; 63: 1881-1894.

32. Chambers JW, LoGrasso PV. Mitochondrial c-Jun N-terminal kinase (JNK) signaling initiates physiological changes resulting in amplification of reactive oxygen species generation. J Biol Chem. 2011; 286: 16052-16062.

33. Lee JS, Lee JJ, Seo JS. HSP70 deficiency results in activation of c-Jun N-terminal Kinase, extracellular signal-regulated kinase, and caspase-3 in hyperosmolarity-induced apoptosis. J Biol Chem. 2005; 280: 6634-6641.

34. Meriin AB, Yaglom JA, Gabai VL, Zon L, Ganiatsas S, Mosser DD, et al. Protein-damaging stresses activate c-Jun N-terminal kinase via inhibition of its dephosphorylation: A novel pathway controlled by HSP72. Mol Cell Biol. 1999; 19: 2547-2555.

35. Mosser DD, Caron AW, Bourget L, Denis-Larose C, Massie B. Role of the human heat shock protein hsp70 in protection against stress-induced apoptosis. Mol Cell Biol. 1997; 17: 53175327.

36. Park HS, Lee JS, Huh SH, Seo JS, Choi EJ. Hsp72 functions as a natural inhibitory protein of c-Jun N-terminal kinase. EMBO J. 2001; 20: 446-456. 
37. Yuan J, Dunn P, Martinus RD. Detection of Hsp60 in saliva and serum from type 2 diabetic and non-diabetic control subjects. Cell Stress Chaperones. 2011; 16: 689-693.

38. Kennedy JW, Hirshman MF, Gervino EV, Ocel JV, Forse RA, Hoenig SJ, et al. Acute exercise induces GLUT4 translocation in skeletal muscle of normal human subjects and subjects with type 2 diabetes. Diabetes. 1999; 48: 1192-1197.

39. Houmard JA, Tanner CJ, Slentz CA, Duscha BD, McCartney JS, Kraus WE. Effect of the volume and intensity of exercise training on insulin sensitivity. J Appl Physiol. 2004; 96: 101-106.

40. Milne KJ, Noble EG. Exercise-induced elevation of HSP70 is intensity dependent. J Appl Physiol. 2002; 93: 561-568.

41. Smolka MB, Zoppi CC, Alves AA, Silveira LR, Marangoni S, Pereira-Da-Silva L, et al. HSP72 as a complementary protection against oxidative stress induced by exercise in the soleus muscle of rats. Am J Physiol Regul Integr Comp Physiol. 2000; 279: R1539-R1545.

42. Liu Y, Mayr S, Opitz-Gress A, Zeller C, Lormes W, Baur S, et al. Human skeletal muscle HSP70 response to training in highly trained rowers. J Appl Physiol. 1999; 86: 101-104.

43. Bajpeyi S, Tanner CJ, Slentz CA, Duscha BD, McCartney JS, Hickner RC, et al. Effect of exercise intensity and volume on persistence of insulin sensitivity during training cessation. J Appl Physiol. 2009; 106: 1079-1085.

44. Kang J, Robertson RJ, Hagberg JM, Kelley DE, Goss FL, Dasilva SG, et al. Effect of exercise intensity on glucose and insulin metabolism in obese individuals and obese NIDDM patients. Diabetes Care. 1996; 19: 341-349.

45. Seals DR, Hagberg JM, Hurley BF, Ehsani AA, Holloszy JO. Effects of endurance training on glucose tolerance and plasma lipid levels in older men and women. JAMA. 1984; 252: 645-649.

46. Rizk NM, Meier DA, Pastorek DJ, Krakower GR, Kissebah AH. Glucose utilization in muscle fiber types: Use of the partial pancreatectomized rat model to distinguish effects of glucose and insulin on insulin resistance. Mol Genet Metab. 1998; 65: 44-50.

47. Barone R, Macaluso F, Sangiorgi C, Campanella C, Marino Gammazza A, Moresi V, et al. Skeletal muscle Heat shock protein 60 increases after endurance training and induces peroxisome proliferator-activated receptor gamma coactivator $1 \alpha 1$ expression. Sci Rep. 2016; 6: 1-18.

48. Holwerda AM, Locke M. Hsp25 and Hsp72 content in rat skeletal muscle following controlled shortening and lengthening contractions. Appl Physiol Nutr Metab. 2014; 39: 1380-1387.

49. Touchberry CD, Gupte AA, Bomhoff GL, Graham ZA, Geiger PC, Gallagher PM. Acute heat stress prior to downhill running may enhance skeletal muscle remodeling. Cell Stress Chaperones. 2012; 17: 693-705.

50. De Filippis E, Alvarez G, Berria R, Cusi K, Everman S, Meyer C, et al. Insulin-resistant muscle is exercise resistant: Evidence for reduced response of nuclear-encoded mitochondrial genes to exercise. Am J Physiol Endocrinol Metab. 2008; 294: E607-E614. 


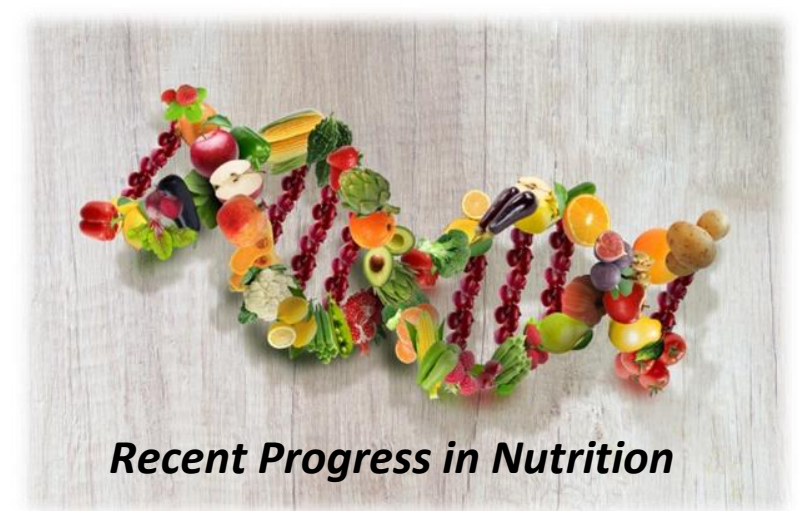

Enjoy Recent Progress in Nutrition by:

1. Submitting a manuscript

2. Joining in volunteer reviewer bank

3. Joining Editorial Board

4. Guest editing a special issue

For more details, please visit:

http://www.lidsen.com/journals/rpn 